\title{
Moderator-variable effect on the Brief Symptom Inventory test-item endorsements of spinal cord injury patients
}

\author{
D G Tate PhD,${ }^{1}$ R K Heinrich MA, ${ }^{1}$ F Maynard MD, ${ }^{1} \mathrm{~S}$ P Buckelew $\mathrm{PhD}^{2}$ \\ ${ }^{1}$ Department of Physical Medicine and Rehabilitation, University of Michigan Medical \\ Center, USA; ${ }^{2}$ Howard Rusk Rehabilitation Institute, University of Missouri Medical \\ School, USA.
}

\begin{abstract}
This study shows that results from the Brief Symptom Inventory (BSI), an instrument used in several US rehabilitation centers to assess psychological distress among patients, are significantly confounded by the somatic characteristics of physically disabled patients with a specific diagnosis: spinal cord injury. Our findings also illustrate the nature and the effect of this moderator variable of physical condition on BSI item endorsement and suggest a method for controlling the moderator's variable effect. The BSI item responses of 288 persons with spinal cord injury (SCI) were compared to a non-patient normative sample of 719 persons. ${ }^{1}$ Thirty-six items reliably differentiated the two groups $(p<0.001)$ and seven of these items were judged to be physical sequelae of SCI. Other highly endorsed items describe a frequent pattern of psychological response to SCI. This response pattern does not appear to reflect the latent variables which are claimed to underlie test items. Expected endorsement of these items by patients with SCI can lead to errors in test interpretation by rehabilitation professionals.
\end{abstract}

Keywords: traumatic spinal cord injury; psychological distress; psychological adjustment; the Brief Symptom Inventory.

\section{Introduction}

The validity of self-report psychological measures depends upon several factors. A most important one is the creation of test relevant norms. In this case, the result of administering the test to a sufficiently large random sample establishes a set of average scores, called norms. Normative scores serve as the reference point for interpretation of subsequent individual administrations of the same test. While validity generalization of a test can be maximized by random sampling a broadly defined population, subpopulations of particular clinical interest are often defined by a very small and specific number of variables which may or may not be adequately represented by this broader normative sample. Further-

Correspondence: Denise G Tate PhD, Assistant Professor, Department of Physical Medicine and Rehabilitation, 1H241 University Hospital, Ann Arbor, Michigan 48109-0050, USA. more, the presence of these variables may affect subjects' tendencies to respond to test items in particular ways. If this is the case, then the normative sample may not be an adequate reference point from which to base scoring interpretations of individuals whose test responses are affected by the presence of variables not included in the normative sample.

This problem is especially relevant when individuals differ on variables such as the presence of physical disability. Myerson ${ }^{2}$ commented in a review of psychological studies of special disabilities that personality tests continued to be interpreted in terms of what the gross scores or configurations are supposed to mean for the nondisabled population. The problem posed by Myerson bears directly on validity generalization; the extent to which those patterns of relationships between assessment input and diagnostic output that have been established for one group are appropriate for another. To 
the extent that these moderator variables affect patients' responses, we can expect to get less accurate interpretation of their responses, thus also placing limits on the test validity generalization.

Taylor $^{3}$ was one of the first persons to examine the effects of spinal cord injury (SCI) as a moderator variable that affected the validity of the Minnesota Multiphasic Personality Inventory (MMPI) for persons with SCI. He found that certain items were consistently endorsed by SCI males with greater frequency than by a matched non-patient sample, and that the content of those items indicated actual physical sequelae of SCI rather than any psychological construct. The endorsement of those somatic items resulted in scoring profiles which reflected significant psychopathology. He suggested that the MMPIs of persons with SCI be scored twice (with and without the somatic items) in order to control for the effects of this moderator variable. These findings were later confirmed using a factor analysis of the items which differentiated SCI subjects from non-SCI subjects. ${ }^{4}$

The Brief Symptom Inventory (BSI), like the MMPI, is a self-report inventory designed to assess overall distress and/or adjustment. In fact, studies on the BSI have used either correlations or profile analyses with the MMPI subscales to establish construct validity of the BSI. ${ }^{5}$ A previous BSI study conducted with SCI patients found that these patients were significantly more distressed in several symptom areas, including somatization, in comparison to the normative non-patient group. ${ }^{6}$

The current study investigates the effects of having SCI upon a person's BSI items responses. Our hypothesis is that SCI is a moderator variable of test responses and that certain BSI items are likely to reflect physical symptoms associated with SCI rather than actual psychological distress. Furthermore, those items which do pertain to psychological status may not be interpretable in terms of the variables (subscales) they are purported to measure. The content of some BSI items may have particular meaning for SCI patients. If this hypothesis is true, then one way to correct this effect and to increase test validity, is to develop a separate set of norms for persons with SCI. While this solution is being addressed elsewhere, ${ }^{7}$ another one is to propose a correction factor to be used by clinicians when interpreting BSI scores of SCI individuals. Like the correction factor for the MMPP, ${ }^{3}$ this factor takes into consideration score differences between the two samples (SCI versus BSI normative sample) on the various test subscales.

\section{Subjects and methods}

The subjects were 288 traumatic SCI patients. All were between the ages of 18 and 65. Patients with psychiatric disorders or major cognitive deficits were excluded from the study. Of this sample, 232 received inpatient rehabilitation and follow up at the University of Michigan Medical Center and 56 at the Howard Rusk Rehabilitation Institute, Columbia, Missouri. The mean age for the sample was 31.33 ( \pm 12.3 years $)$ while the average education was 12 th grade. The mean time since injury was 14.5 months, ranging from discharge to 6 years. Most patients were males $(80.2 \%)$ and single $(64.3 \%)$. Forty eight percent of the subjects were diagnosed as having paraplegia and $52 \%$ quadriplegia. The neurological level of the SCI was not available on 15 patients $(5.2 \%)$.

Psychological distress, broadly defined as a transient affective reaction to stressful external events, has been identified as the single most important indicator of psychological adjustment post SCI. ${ }^{8}$ The BSI was used to assess psychological distress in the sample. The BSI is an abbreviated version of the Symptom Checklist $90^{9}$ containing 53 items distributed across nine subscales of distress (somatization; obsessive-compulsive; interpersonal sensitivity; depression; anxiety; hostility; phobic anxiety; paranoid ideation; and psychoticism). Patients are asked to rate how much each symptom or problem has been distressful during the past week, using a Likert-type scale. BSI scores reflect emotional states and are not intended to measure enduring individual traits. The test also provides these global indices of distress and psychological dysfunction: the total number of symptoms 
(PST); average intensity of positive symptoms (PSDI), and average item response (GSI).

Do patients with SCI respond differently to the BSI in comparison to those without SCI? To answer this question the patient mean profile was compared with the nonSCI normative group using Student's $t$ tests for independent samples and Bonferroniadjusted probabilities for multiple tests. The normative sample ${ }^{1}$ had a mean age of 46 ( \pm 14.2 years). Males comprised $50.7 \%$ of this sample while $49.3 \%$ were females. Eighty-four percent $(84.6 \%)$ were either single or married (specific data on single versus married was not available), the other $15.4 \%$ included widows or divorced individuals. Differences by gender and neurological classification among SCI patients were also determined by using Student's $t$ tests. First, a Bonferroni correction for adjusted probabilities was calculated for statistical analyses involving multiple tests. ${ }^{10}$ Second, for each of the 53 items the response distribution of the SCI sample was compared to the distribution of responses of the original non-patient normative sample. Since these item responses were not normally distributed, and because only frequency distributions are available for the original norm group (Derogatis, personal communication), $\chi^{2}$ tests for independence were used to make these comparisons. In order to maximize the specificity of these tests we selected a stringent Alpha of 0.001 $\left(\chi^{2}=27.09\right)$. Third, all BSI items were independently examined by a panel of experienced SCI rehabilitation personnel (two attending physicians, one nurse, and three psychologists). They were asked to check BSI items which reflected physical sequelae of SCI. Items for which at least four of the six judges were in agreement were labeled somatically relevant. The Global Severity Index (GSI) for each subject was recalculated excluding these somatic items. The corrected mean BSI/GSI score for the group was then compared with the uncorrected mean BSI/GSI score using Student's paired comparisons $t$ test.

\section{Results}

When first comparing BSI scores across the two samples, subjects with SCI reported significantly higher levels of distress than non-SCI subjects (Table I). Differences

Table 1 Comparison of mean BSI scores of SCI and non-SCI samples

\begin{tabular}{lcccc}
\hline BSI & \multicolumn{2}{c}{ SCI } & & \multicolumn{2}{c}{$\begin{array}{c}\text { Non-patient } \\
(n=288)\end{array}$} & Mean & SD \\
\cline { 2 - 3 } & Mean & SD & & \\
\hline Subscales & & & & \\
SOM & $0.78^{\mathrm{a}}$ & 0.57 & 0.29 & 0.40 \\
OB-COM & $0.77^{\mathrm{a}}$ & 0.64 & 0.43 & 0.48 \\
INT-SEN & $0.46^{\mathrm{b}}$ & 0.66 & 0.32 & 0.48 \\
DEP & $0.62^{\mathrm{a}}$ & 0.70 & 0.28 & 0.46 \\
ANX & $0.52^{\mathrm{b}}$ & 0.56 & 0.35 & 0.45 \\
HOS & $0.53^{\mathrm{b}}$ & 0.63 & 0.35 & 0.42 \\
PHOB & $0.40^{\mathrm{b}}$ & 0.57 & 0.17 & 0.36 \\
PAR & $0.60^{\mathrm{b}}$ & 0.69 & 0.34 & 0.45 \\
PSY & $0.37^{\mathrm{b}}$ & 0.58 & 0.15 & 0.31 \\
Global indices & & & & \\
GSI & $0.57^{\mathrm{b}}$ & 0.48 & 0.30 & 0.31 \\
PSDI & $1.78^{\mathrm{b}}$ & 0.62 & 1.29 & 9.41 \\
PST & $16.55^{\mathrm{b}}$ & 11.37 & 11.45 & -29 \\
\hline
\end{tabular}

${ }^{\mathrm{a}} p<0.0003,{ }^{\mathrm{b}} p<0.001$.

Norms provided by Derogatis \& Spencer, 1982. ${ }^{1}$

These values were also significant when using Bonferroni $t$ statistics $(t \geqslant 3.36$; Alpha $=0.01)$. 
were observed on all BSI subscales and global indices with the most significant findings occurring in somatization, obsessive-compulsive, and depression subscales. In comparison to non-SCI subjects, SCI subjects reported more symptoms (PST) and the intensity of their reported symptoms (PSDI) was much higher. These findings significantly contributed to the elevation of their global severity distress index (GSI). There were no gender differences in the SCI sample, with the exception of the somatization subscale. Women with SCI reported slightly greater somatic distress than SCI men $(p<0.05)$. With respect to SCI neurological classification, there were no differences between distress reported by patients with paraplegia versus those with quadriplegia.

The null hypothesis for item response distributions being independent of the sample from which the responses were taken was rejected for 36 of the 53 BSI items (Alpha $=0.001$; Table II). The 36 items in Table II are listed in descending order of $\chi^{2}$ values. Thus items near the top of this list were endorsed with greater frequency and intensity by persons in this sample. In this sense, these items were more relevant and meaningful for this group.

Two items had $100 \%$ agreement among all six judges as reflective of physical symptoms of SCI: numbness and tingling in parts of your body (item 33) and feeling weak in parts of your body (item 37) were expected physical consequences of SCI. Five of the six judges $(83 \%)$ agreed on hot or cold spells (item 30), while four of the six judges (66\%) concurred on symptoms of poor appetite (item 11), trouble falling asleep (item 25), nausea or upset stomach (item 23), and faintness or dizziness (item 2). There was general agreement among the judges that these last four items are likely to be intensified during inpatient treatment of SCI due to high levels of medications and the effects of an unfamiliar hospital environment. Five of the selected seven items occur on the BSI somatization subscale, while the remaining two $(11,25)$ additional items are not part of any BSI ideation subscale. The difference between the recalculated BSI/GSI, correcting for the somatically relevant items, and the BSI/GSI without such correction was statistically significant $(p<0.001$; Table III), thus suggesting that inclusion of somatically relevant items on the BSI tends to artificially elevate global distress scores. This corrected BSI/GSI mean score was still significantly greater than the original mean score for the non-SCI norm group $(p<0.001)$.

\section{Discussion}

The main aim of the present study was to determine whether SCI has a moderator effect on BSI responses of patients with this diagnosis. $\chi^{2}$ analyses identified a set of symptom responses which uniquely describe the SCI sample in relation to the non-SCI sample. Seven of these items were judged to be likely physical consequences of SCI. According to the BSI, five of these items measure somatization, a construct defined by perceptions of distress originating either in actual physical disease or in bodily discomfort and serving primarily a psychological function. Self-reported physical complaints have often been associated with neuroticism in previous health research. ${ }^{11,12}$ For most SCI persons, the somatically relevant items of the BSI do not serve a psychological function, and valid interpretations of their BSI scores must take this into account.

The removal of seven SCI somatic-related BSI items resulted in a statistically significant decrease in global measures of psychological distress. Yet persons with SCI report significantly higher levels of psychological distress than do non-SCI persons, even when the physical consequences of the injury are controlled for. While we expected that somatically relevant items would have made a greater contribution to overall distress, this finding underscores the traumatic nature of SCI and its impact upon multiple domains of functioning.

What is the nature of the psychological distress experienced by persons with SCI? A number of BSI items (e.g. 15, 38, 49, 51, 42) relate to the psychological experience of SCI defined by loss of bodily functions, loss of life control, having conflicting expectations about recovery, and an overall sense of 
Table II $\chi^{2}$ test for independence between the SCI sample and the non-patient sample on BSI items

\begin{tabular}{|c|c|c|c|}
\hline Item & Content & Subscale & $\chi^{2 \mathrm{a}}$ \\
\hline 33 & Numbness or tingling in parts of your body & SOM & 414.2 \\
\hline 37 & Feeling weak in parts of your body & SOM & 356 \\
\hline 15 & Feeling blocked in getting things done & OB-COM & 291.3 \\
\hline 38 & Feeling tense or keyed up & ANX & 245.5 \\
\hline 49 & Feeling so restless you cannot sit still & ANX & 221.5 \\
\hline 51 & Feeling that people will take advantage of you & PAR & 178.4 \\
\hline 42 & Feeling very self conscious with others & INT-SEN & 162.3 \\
\hline 30 & Hot or cold spells & SOM & 132.1 \\
\hline 17 & Feeling blue & DEP & 114.4 \\
\hline 35 & Feeling hopeless about the future & DEP & 105.6 \\
\hline 27 & Difficulty making decisions & $\mathrm{OB}-\mathrm{COM}$ & 97.8 \\
\hline 11 & Poor appetite & b & 96.0 \\
\hline 25 & Trouble falling asleep & b & 86.1 \\
\hline 43 & Feeling uneasy in crowds & PHOB & 77.0 \\
\hline 18 & Feeling no interest in things & DEP & 73.7 \\
\hline 46 & Getting into frequent arguments & HOS & 64.2 \\
\hline 20 & Your feelings being easily hurt & INT-SEN & 57.5 \\
\hline 9 & Thoughts of ending your life & DEP & 56.7 \\
\hline 23 & Nausea or upset stomach & SOM & 51.2 \\
\hline 22 & Feeling inferior to others & INT-SEN & 50.6 \\
\hline 10 & Feeling that most people cannot be trusted & PAR & 48.4 \\
\hline 44 & Never feeling close to another person & PSY & 46.0 \\
\hline 48 & Others not giving you proper credit for your achievements & PAR & 43.7 \\
\hline 26 & Having to check and and double check what you do & OB-COM & 42.7 \\
\hline 39 & Thoughts of death or dying & $\mathrm{b}$ & 42.0 \\
\hline 32 & Your mind going blank & OB-COM & 40.0 \\
\hline 40 & Having urges to beat, injure, or harm someone & HOS & 36.6 \\
\hline 31 & $\begin{array}{l}\text { Having to avoid certain things, places, or activities because } \\
\text { they frighten you }\end{array}$ & PHOB & 36.0 \\
\hline 16 & Feeling lonely & DEP & 35.6 \\
\hline 53 & The idea that something is wrong with your mind & PSY & 35.0 \\
\hline 24 & Feeling that you are watched or talked about by others & PAR & 34.4 \\
\hline 28 & Feeling afraid to travel on buses, subways, or trains & PHOB & 31.1 \\
\hline 12 & Suddenly scared for no reason & ANX & 30.7 \\
\hline 2 & Faintness or dizziness & SOM & 30.7 \\
\hline 6 & Feeling easily annoyed or irritated & HOS & 28.7 \\
\hline 1 & Nervousness or shakiness inside & ANX & 27.3 \\
\hline
\end{tabular}

${ }^{a}$ Critical value of $\chi^{2}=27.09(\mathrm{df}=4$; Bonferroni adjusted Alpha $=0.001)$

bdenotes items which do not load on any subscale.

Table III Comparisons of corrected and uncorrected $\mathrm{BSI} / \mathrm{GSI}$ mean scores of SCI sample and to BSI/GSI mean score of non-patient sample

\begin{tabular}{lll}
\hline SCI corrected BSI/GSI & $0.52^{\mathrm{a}}$ & $(1)$ \\
SCI uncorrected BSI/GSI & $0.57^{\mathrm{a}}$ & $(2)$ \\
Non-patient BSI/GSI & $0.30^{\mathrm{a}}$ & $(3)$ \\
\hline
\end{tabular}

${ }^{a} p<0.001$ for paired means (1), (2) and nonpaired means (1), (3) and (2), (3). self devaluation associated with increased unwanted dependency on others. Without being exhaustive, a closer examination of those items which were most meaningful to persons with SCI, i.e. items endorsed with greatest frequency and intensity, is helpful in understanding a frequent (though not universal) pattern of psychological response to SCI. 
Items 38 (feeling tense and keyed up) and 49 (feeling so restless you cannot sit still) are not direct physical symptoms of SCI, yet they do reflect somatic experience of anxiety. These symptoms can be attributed to an interaction of several factors, including experiencing pain and discomfort, medication side effects, separation or loss of significant others, lack of physical accessibility to the environment, and continued worries about future physical and financial wellbeing. Severe restrictions in mobility may particularly lead SCI patients to intense feelings of restlessness simply due to the inaccessibility of usual channels for physical discharge of tension. Item 42 (feeling very self conscious with others) is part of the interpersonal sensitivity subscale of the BSI, while item 51 (feeling that people will take advantage of you) is part of the BSI paranoid ideation subscale. Both items identify distress in the interpersonal domain for persons with SCI. Significant changes in body image, in capacity for self care and mobility can result in abnormal levels of self consciousness and distrust of others.

For some endorsed items, the underlying psychological construct is questionable. In particular, items 15 (feeling blocked in getting things done) and 27 (difficulty making decisions) have been conceptually linked by the test authors to characteristics of obsessive-compulsiveness. Yet for persons with SCI, positive responses to item 15 can be interpreted as appropriate in view of their current physical limitations related to SCI. Endorsing item 27 might also be a reasonable response to a dramatically altered life situation or a correlate of perceiving few available life options. Careful confirmatory factor-analytic work is needed to examine this question of response appropriateness and for empirical derivation of potentially more meaningful BSI scales, such as has already been accomplished within a rehabilitation sample of persons experiencing chronic pain. ${ }^{13,14}$

Contrary to previous suggestions for separate BSI norms for males and females ${ }^{1.15-17}$ our findings showed gender differences only in the somatic items. Expected gender differences on self-report inventories may be due, in part, to a defensive response set stemming from normative cultural ideals of masculinity where the expression of intense feelings or distress by males is viewed as unacceptable. Among our sample, the intensity of distress associated with the experience of the psychosocial aspects of SCI may have been great enough to overcome this usual response bias.

Statistically significant differences on BSI items were not found when comparing responses from persons with quadriplegia with those with paraplegia. This finding suggests that severity of SCI was not associated with the experience of greater or lesser distress in the context of this study.

In summary, our results suggest that BSI interpretation can be significantly affected by one's diagnosis of SCI. More specifically, caution needs to be applied when using the BSI to assess distress among persons with SCI. We propose a two-prong approach to address this problem: first, using BSI/SCI relevant norms for a more accurate interpretation of distress among persons with SCI; and second, scoring the test with and without the seven somatically relevant items. This method of correcting for the physical health moderator follows the approach suggested earlier ${ }^{3}$ when addressing this same issue with the MMPI. The problem with this second approach is that it could lead to an overcorrection. It is impossible to determine exactly how much the response reflects distress associated with actual SCI symptomatology versus premorbid personality traits which could equally account for the intensity of distress reported. In addition, neurologically intact populations occasionally respond in the abnormal direction on each item while some persons with SCI do not respond positively to items associated with physical sequelae of SCI.

A preferred approach seems to be the use of relevant norms to evaluate overall distress in relation to other SCI persons. ${ }^{7}$ If, after comparing a patient's score with the relevant norms, distress is still found to be abnormally elevated, the clinician is advised to examine the subject's individual item responses. This method would allow the clinician to obtain a sense of the subject's personal adjustment to the various aspects of the injury. Although this study focused 
on the effect of SCI as the moderator variable, it is likely that other physically disabled populations would encounter similar problems in relation to the interpretation of their BSI scores. These facts make the need to account for these specific effects even more important if one is to find valid and reliable instruments to use in clinical services and research applied to people with disabilities.

\section{References}

1 Derogatis LR, Spencer MS (1982) The Brief Symptom Inventory (BSI) Administration, Scoring, and Procedures Manual I. John Hopkins Univ. School of Medicine, Baltimore: 4.

2 Myerson L (1957) Special disabilities. Annu Rev Psychol 8: 437-467.

3 Taylor GP (1970) Moderator-variable effect on personality test-item endorsements of physically disabled patients. J Consult Clin Psychol 35: 183-188.

4 Kendall PC, Edinger J, Eberly C (1978) Taylor's MMPI correction factor for spinal cord injury: Empirical Endorsement. J Consult Clin Psychol 46: 370-371.

5 Derogatis LR, Melisaratos N (1983) The Brief Symptom Inventory: An introductory report. Psychol Med 13: 595-605.

6 Tate DG, Kewman DG, Maynard F (1990) The Brief Symptom Inventory: Measuring psychological distress in spinal cord injury. Rehabil Psychol 35: 211-216.

7 Heinrich RK, Tate DG, Buckelew SP (1994) Brief Symptom Inventory norms for spinal cord injury. Rehabil Psychol. 39.

8 Shadish WR, Hickman D, Arrick MC (1981) Psychological problems of spinal cord injury patients: Emotional distress as function of time and locus of control J Consult Clin Psychol 49: 297-318.

9 Derogatis LR (1977) The SCL-90 Manual I: Scoring, Administration and Procedures for the SCL-90. John Hopkins University School of Medicine, Baltimore: 4.

10 Wilkinson L, Hill MA, Vang E (1992) SYSTAT: Statistics, Version 5.2 Edition. SYSTAT Inc, Evanston, IL: 724.

11 Costa PT, McCrae RR (1987) Neuroticism, somatic complaints, and disease: Is the bark worse than the bite? J Pers 55: 299-316.

12 Watson D, Pennebaker JW (1989) Health complaints, stress, and distress: Exploring the central role of negative affectivity. Psychol Rev 96: 234-254.

13 Shutty MS, DeGood DE, Schwartz DP (1986) Psychological dimensions of distress in chronic pain patients: A factor analytic study of Symptom Checklist-90 responses. J Consult Clin Psychol 54: 836-842.

14 Shutty MS, DeGood DE (1987) Cluster analyses of responses of low-back pain patients to the SCL-90: Comparison of empirical versus rationally derived subscales. Rehabil Psychol 32: 133-144.

15 Cochran CD, Hale WD (1985) College student norms on the Brief Sympton Inventory. Clin Psychol 41: 777-779.

16 Francis VM, Rajan P, Turner N (1990) British community norms for the Brief Symptom Inventory. Br J Clin Psychol 29: 115-116.

17 Hale WD, Cochran CD, Hedgepeth BE (1984) Norms for the elderly on the Brief Symptom Inventory. J Consult Clin Psychol 52: 321-322. 